\title{
Insulin resistance characterizes glucose uptake in skeletal muscle but not in the heart in NIDDM
}

\author{
T. Utriainen ${ }^{1,5}$, T. Takala ${ }^{1}$, M. Luotolahti ${ }^{2}$, T. Rönnemaa ${ }^{3}$, H. Laine ${ }^{1}$, U. Ruotsalainen ${ }^{1}$, M. Haaparanta ${ }^{4}$, \\ P. Nuutila ${ }^{3}$, H. Yki-Järvinen ${ }^{5}$ \\ ${ }^{1}$ Turku PET Centre, University of Turku, Turku, Finland \\ ${ }^{2}$ Department of Clinical Physiology, University of Turku, Turku, Finland \\ ${ }^{3}$ Department of Medicine, University of Turku, Turku, Finland \\ ${ }^{4}$ Radiopharmaceutical Chemistry Laboratory, University of Turku, Turku, Finland \\ ${ }^{5}$ Department of Medicine, Division of Endocrinology and Diabetology, University of Helsinki, Helsinki, Finland
}

\begin{abstract}
Summary Skeletal muscle insulin resistance and coronary heart disease (CHD) often precede non-insulin-dependent diabetes mellitus (NIDDM). A recent study showed the myocardium of patients with CHD to be insulin resistant, independent of blood flow. We determined whether myocardial insulin resistance is a feature of NIDDM patients with no CHD. Skeletal muscle and myocardial glucose uptake were determined in 10 patients with NIDDM and 9 ageand weight-matched normal men of similar age and body mass index men using $\left[{ }^{18} \mathrm{~F}\right]$-2-fluoro-2-deoxy-Dglucose and positron emission tomography under normoglycaemic hyperinsulinaemic conditions. Whole body glucose uptake, as determined by the euglycaemic clamp technique, was significantly lower in the patients with NIDDM $(35 \pm 3 \mu \mathrm{mol} / \mathrm{kg}$ body weight $\cdot \min )$ than the normal subjects $(45 \pm 3 \mu \mathrm{mol} /$ $\mathrm{kg}$ body weight $\cdot \min , p<0.02)$. Insulin-stimulated femoral muscle glucose uptake was significantly lower in the patients with NIDDM $(71 \pm 6 \mu \mathrm{mol} / \mathrm{kg}$ mus-
\end{abstract}

cle $\cdot \min )$ than in the normal subjects $(96 \pm 5 \mu \mathrm{mol} /$ $\mathrm{kg}$ muscle $\cdot \min , p<0.01)$. Whole body glucose uptake was correlated with femoral muscle glucose uptake in the entire group $(r=0.76, p<0.001)$, in patients with NIDDM and in normal subjects. Rates of insulin-stimulated myocardial glucose uptake were comparable between the patients with NIDDM $(814 \pm 76 \mu \mathrm{mol} / \mathrm{kg}$ muscle $\cdot \min )$ and the normal subjects $(731 \pm 63 \mu \mathrm{mol} / \mathrm{kg}$ muscle $\cdot \min , \quad p>0.4)$. Whole body or femoral muscle, and myocardial glucose uptake were not correlated in all subjects, patients with NIDDM or normal subjects. We conclude that insulin resistance of the myocardium is not a feature of uncomplicated NIDDM. [Diabetologia (1998) 41: 555-559]

Keywords Myocardium, insulin resistance, non-insulin-dependent diabetes mellitus, positron emission tomography.
Signs or symptoms of macrovascular disease are frequently found even prior to the development of noninsulin-dependent diabetes mellitus (NIDDM) [1]. This suggests that coronary heart disease (CHD)

Received: 8 August 1997 and in revised form: 6 December 1997

Corresponding author: H. Yki-Järvinen, M.D., Department of Medicine, Division of Endocrinology and Diabetology, University of Helsinki, Haartmaninkatu 4, FIN-00290 Helsinki, Finland

Abbreviations: NIDDM, Non-insulin-dependent diabetes mellitus; CHD, coronary heart disease; FDG, $\left[{ }^{18} \mathrm{~F}\right]$-2-fluoro-2deoxy-D-glucose; PET, positron emission tomography. and NIDDM share a pathophysiologic feature, which predisposes to both diseases. Classic risk factors such as serum cholesterol, age, gender, hypertension and smoking explain only a fraction of the $2-4$-fold increase in cardiovascular mortality in patients with NIDDM [1, 2]. Insulin resistance, usually measured by quantitating the rate of insulin-stimulated glucose uptake, qualifies as the underlying pathophysiologic abnormality for both CHD and NIDDM, since it predicts, independent of other risk factors, both disorders $[3,4]$.

The mechanisms linking insulin resistance to CHD are unclear but could involve lipid abnormalities such as an increase in the concentration of atherogenic small dense LDL particles, which accompanies insu- 
lin resistance and hypertriglyceridaemia [5]. On the other hand, insulin resistance has been found to predict CHD even independent of lipid changes [6]. Recently, a defect in the ability of insulin to stimulate myocardial glucose uptake was demonstrated in both non-diabetic [7] and diabetic [8] patients with CHD. This novel defect has been suggested to contribute to, or reflect defects in glycolysis, which characterize heart failure in animal models [9], and might limit utilization of glucose under postprandial and ischaemic conditions [9]. It is, however, unknown, whether insulin resistance in the heart is a cause or a consequence of impaired cardiac function. This question can be answered by quantitating insulin sensitivity simultaneously in both skeletal muscle and the heart in patients who have NIDDM but no CHD. This was the aim of the present study, in which we quantitated heart and skeletal muscle glucose uptake using $\left[{ }^{18} \mathrm{~F}\right]$-2-fluoro-2-deoxy-D-glucose ( $\left.\left[{ }^{18} \mathrm{~F}\right] \mathrm{FDG}\right)$ and positron emission tomography (PET) under normoglycaemic hyperinsulinaemic conditions in patients with NIDDM but no CHD and in non-diabetic subjects.

\section{Subjects and methods}

Subjects. Ten male patients with NIDDM (age $43 \pm 2$ years, body mass index $27.3 \pm 0.7 \mathrm{~kg} / \mathrm{m}^{2}$, blood pressure $126 \pm 4$ / $84 \pm 4 \mathrm{mmHg}$, glycosylated hemoglobin $\mathrm{A}_{1 \mathrm{c}} 8.1 \pm 0.5 \%$ ) and 9 normal men (45 \pm 3 years, $26.4 \pm 0.7 \mathrm{~kg} / \mathrm{m}^{2}, \quad 127 \pm 4 / 82 \pm$ $2 \mathrm{mmHg}, 5.4 \pm 0.3 \%$ ) volunteered for the studies. The patients did not have clinical or biochemical evidence of diseases other than NIDDM and had no signs of micro- or macrovascular disease. Coronary artery disease was excluded by a normal resting ECG and by a work-conducted maximal bicycle exercise test. Four patients were treated with diet alone while 6 were treated with sulfonylureas and/or metformin. Oral antidiabetic drugs were discontinued for 2 days prior to the studies. The duration of NIDDM averaged $3 \pm 1$ years. The normal subjects were healthy as judged by history, physical examination and routine laboratory tests, and were not taking any medication. Written informed consent was obtained after the purpose, nature and potential risks were explained to the subjects. The experimental protocol was reviewed and approved by the ethical committee of the Turku University Central Hospital.

Study design. All subjects were studied after a 10-12 h overnight fast. Two catheters were inserted, one in an antecubital vein for infusion of glucose and insulin and injection of $\left[{ }^{18} \mathrm{~F}\right] \mathrm{FDG}$, and another in the opposite radial or brachial artery for blood sampling. The patients with NIDDM were rendered normoglycaemic before the start of the study by using a lowdose insulin infusion according to the algorithm described by Mokan and Gerich [10]. Whole body, skeletal and heart muscle glucose uptake rates were thereafter determined by combining the euglcaemic hyperinsulinaemic clamp technique with $\left[{ }^{18} \mathrm{~F}\right]$ FDG and PET, as described in detail below.

Whole body glucose uptake. Whole body glucose uptake was quantitated using the euglcaemic hyperinsulinaemic clamp technique [11]. Serum insulin was increased for $180 \mathrm{~min}$ using a primed-continuous $(30 \mathrm{pmol} / \mathrm{kg} \cdot \mathrm{min})$ infusion of insulin
(Velosulin; Novo Nordisk A/S, Bagsvaerd, Denmark). Normoglycaemia was maintained using an infusion of $20 \%$ glucose based on frequent arterial plasma glucose measurements. Whole body glucose uptake was calculated from the glucose infusion rate during the period of PET scanning (60-160 min). Serum free insulin concentrations were measured every $30 \mathrm{~min}$ by radioimmunoassay [12].

Heart and skeletal muscle glucose uptake. $\left[{ }^{18} \mathrm{~F}\right] \mathrm{FDG}\left(T_{1 / 2}=\right.$ $109.8 \mathrm{~min}$ ) was synthesized with an automatic apparatus [12]. Approximately $5 \mathrm{mCi}(185 \mathrm{MBq})$ of $\left[{ }^{18} \mathrm{~F}\right] \mathrm{FDG}$ was injected intravenously over $2 \mathrm{~min}$. A dynamic scan of the femoral region was performed for 90 min and continued with a dynamic scan of the thoracic region for $18 \mathrm{~min}$. Plasma radioactivity was measured with an automatic gamma counter (Wizard 1480 3"; Wallac, Turku, Finland). An eight-ring ECAT 931/08-tomograph (Siemens/CTI Corp., Knoxville, Tenn., USA) with an axial resolution of $6.7 \mathrm{~mm}$, in plane resolution of $6.5 \mathrm{~mm}$, and final inplane resolution of $8 \mathrm{~mm}$, was used. Before the emission scan, a transmission scan was performed as previously described [12]. All data were corrected for deadtime, decay and measured photon attenuation. Dynamic FDG scans were reconstructed into a $128 \times 128$ matrix using a Hann-filter with a cut-off frequency of 0.5 . Regions of interest were drawn within femoral muscles as previously described [12]. For determination of myocardial glucose uptake, large regions of interest were drawn on at least four representative midventricular slices avoiding myocardial borders. All myocardial time-activity curves were corrected for partial volume effect and spillover from cavity [13]. The graphical analysis derived from the three compartment model of $\left[{ }^{18} \mathrm{~F}\right] \mathrm{FDG}$ kinetics as described previously was used to quantitate the fractional rate of tracer phosphorylation $K_{\mathrm{i}}[12]$.

Echocardiography. M-mode and two-dimensional echocardiography (Aloca SSD-870; Aloca Co. Ltd., Tokyo, Japan) were performed, and Penn-cube left ventricular mass was calculated as previously described $[14,15]$.

Statistical analysis. Group comparisons were performed with the unpaired Student's $t$-test for normally distributed data, and correlations were calculated using Pearson's correlation analysis. The results are expressed as mean \pm SEM.

\section{Results}

Whole body glucose uptake. Fasting plasma glucose ( $8.3 \pm$ vs $5.4 \pm \mathrm{mmol} / \mathrm{l}$, NIDDM vs normal subjects, $p<0.001)$ and serum free insulin concentrations (73 \pm 24 vs $36 \pm 6 \mathrm{pmol} / \mathrm{l}$, respectively, $p<0.05$ ), were higher in patients with NIDDM than in normal subjects. During hyperinsulinaemia, serum free insulin concentrations averaged $2434 \pm 153 \mathrm{pmol} / 1$ in patients with NIDDM and $2666 \pm 79 \mathrm{pmol} / \mathrm{l}$ in the normal subjects (NS), and plasma glucose concentrations were $5.3 \pm 0.1$ and $5.4 \pm 0.1 \mathrm{mmol} / 1$, respectively (NS). Serum non-esterified fatty acid (NEFA) concentrations were not significantly different between the groups $(205 \pm 18$ vs $155 \pm 20 \mu \mathrm{mol} / \mathrm{l}$, NS). Whole body glucose uptake during hyperinsulinaemia was significantly lower in the patients with NIDDM $(35 \pm 3 \mu \mathrm{mol} / \mathrm{kg}$ body weight $\cdot \min )$ than the normal subjects $(45 \pm 3 \mu \mathrm{mol} / \mathrm{kg}$ body weight $\cdot \min , p<0.02)$. 

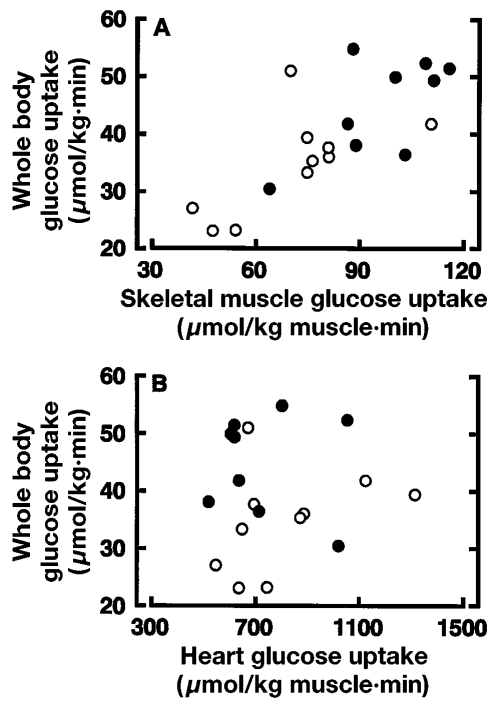

Fig. 1 A, B. Interrelationships between femoral muscle and whole body glucose uptake $(\mathbf{A})$, and heart and whole body glucose uptake $(\mathbf{B})$ in the patients with NIDDM $(\bigcirc)$ and in normal subjects

Skeletal muscle and heart glucose uptake. Insulinstimulated femoral muscle glucose uptake was significantly lower in the patients with NIDDM $(71 \pm$ $6 \mu \mathrm{mol} / \mathrm{kg}$ muscle $\cdot \min )$ than in the normal subjects $(96 \pm 5 \mu \mathrm{mol} / \mathrm{kg}$ muscle $\cdot \min , p<0.01)$. Whole body glucose uptake was correlated with femoral muscle glucose uptake in the entire group $(r=0.76, p<$ $0.001)$, in patients with NIDDM $(r=0.66, p<0.05)$ and in normal subjects $(r=0.66, p<0.05)$ (Fig. 1$)$.

Left ventricular masses were similar between patients with NIDDM (222 $\pm 8 \mathrm{~g})$ and the normal subjects $(223 \pm 11 \mathrm{~g}, \mathrm{NS})$. Rates of insulin-stimulated myocardial glucose uptake were comparable between the patients with NIDDM $(814 \pm 76 \mu \mathrm{mol} / \mathrm{kg}$ muscle $\cdot$ $\mathrm{min})$ and the normal subjects $(731 \pm 63 \mu \mathrm{mol} / \mathrm{kg}$ muscle $\cdot \min , p>0.4)$. Rate-pressure products were comparable during hyperinsulinaemia in patients with NIDDM [9228 $\pm 684 \mathrm{mmHg} \cdot$ (beats/min)] and the normal subjects $(8380 \pm 596)$. There was no significant correlation between whole body and myocardial glucose uptake in all subjects $(r=0.06, \mathrm{NS})$, patients with NIDDM $(r=0.37$, NS) or normal subjects $(r=-0.08$, NS) (Fig. 1), or between femoral muscle and myocardial glucose uptake (data not shown).

\section{Discussion}

In the present study, we quantitated skeletal muscle and heart glucose uptake in patients with NIDDM who had no evidence of CHD clinically or based on a maximal bicycle exercise test. We found insulin resistance at the level of the whole body, the magnitude of which was closely related to that in skeletal muscle. Rates of insulin-stimulated heart glucose uptake, were, however, comparable in patients with NIDDM and normal subjects. These data indicate that patients with NIDDM do not exhibit generalized insulin resistance, and that the insulin resistance which has been found in non-diabetic [7] and NIDDM [8] subjects with CHD is likely to be a consequence of neurohormonal or other alterations secondary to heart failure or CHD and not NIDDM.

Use of $\left[{ }^{18} \mathrm{~F}\right] \mathrm{FDG}$ as a tracer to quantitate glucose uptake assumes that this tracer reflects the behaviour of the tracee. A lumped constant term is used to adjust for differences between the tracer and the tracee. The lumped constant has been found to be unaffected by insulin $(5000 \mathrm{mU} / \mathrm{l})$ in the isolated arterially perfused rabbit interventricular septum [16], and by 20fold differences in glucose metabolic rates in vivo in the heart of anaesthetized dogs [17]. In the isolated perfused working rat heart, however, Hariharan et al. [18], found insulin (1000 mU/l) to increase glucose but not $\left[{ }^{18} \mathrm{~F}\right] \mathrm{FDG}$ uptake. The latter result differs from effects of insulin on FDG uptake in the human heart in vivo, in which mean glucose uptake rates, increase approximately $12-34$-fold by physiological insulin concentrations $[19,20]$. Even at low (NEFA) concentrations, FDG-uptake increases 2fold by insulin in humans [20]. The latter data are similar to those reported in catheterization studies [21] and support, together with several additional in vivo observations (vide infra) the view that glucose uptake measured with FDG and PET provides information which is fully compatible with physiological knowledge. Similarly, concentrations of plasma NEFA are inversely correlated with rates of FDG uptake [12] and arterial-coronary sinus glucose differences [22]. Several indices of cardiac work are also directly correlated with glucose uptake under normoglycaemic hyperinsulinaemic conditions $[12,14,23]$ where glucose is the main fuel for heart energy production.

The present data are consistent with data from previous studies examining insulin sensitivity of heart glucose uptake in various groups differing with respect to their muscle insulin sensitivity. Patients with IDDM $[23,24]$ with no CHD have insulin resistance in skeletal muscle but no alteration in cardiac work or myocardial glucose uptake under insulin-stimulated conditions. In contrast, in athletes with a decrease in cardiac work per heart mass, insulin-stimulated glucose uptake in the heart is diminished, while peripheral insulin sensitivity is enhanced [14]. In patients with essential hypertension, the opposite picture emerges: heart glucose uptake and cardiac work per unit heart mass are increased in proportion to the elevation of blood pressure, while peripheral insulin sensitivity is decreased [25]. Thus, if changes in insulin sensitivity are associated with alterations in cardiac work (hypertension, physical fitness), heart glucose uptake is altered in proportion to the altera- 
tion in cardiac work under insulin-stimulated conditions. If peripheral insulin resistance is not associated with alterations in cardiac work, insulin-stimulated glucose uptake appears normal. The study of $\mathrm{Pa}$ ternostro et al. [7] who found insulin resistance in the myocardium, fits in neither of these categories as 10 of the patients with CHD were classified as NYHA class III and 5 as NYHA class II, and all had symptoms of ischaemic heart disease. The finding of insulin resistance in the myocardium of such patients, even in segments with normal wall motion and blood flow [7], could be due to factors such as increased norepinephrine [26, 27] or NEFA concentrations [27], which accompany heart failure. In contrast to the present data, Voipio-Pulkki et al. [8] found NID$\mathrm{DM}$ to be associated with cardiac insulin resistance in patients with CHD. Although one could argue that NIDDM influences myocardial glucose uptake differently in patients with and without CHD, other explanations are more likely to underlie the discrepant findings. First, in the study of Voipio-Pulkki et al. [8] the patients with NIDDM were studied under hyperglycaemic conditions and the control subjects under normoglycaemic conditions [8]. In the rat heart, the glucose concentration profoundly affects the rate-limiting step for glucose utilization and consequently the lumped constant [28]. Whether this also applies in humans is currently unknown. Another factor to consider is that we used a supramaximal insulin concentration which resulted in comparable suppression of NEFA concentrations in both groups. Since lipolysis is exquisitely sensitive to insulin [29] and since the antilipolytic effect of insulin may be altered by NIDDM or obesity [30], the present data do not exclude the existence of myocardial insulin resistance under conditions where myocardial glucose uptake is dependent upon plasma NEFA availability $[12,31]$. Also, the present data do not exclude the possibility that older or more obese patients or patients with longer duration of NIDDM would be characterized by cardiac insulin resistance.

We conclude that insulin resistance in the myocardium is not an inherent feature of uncomplicated NIDDM. Impaired myocardial glucose utilization is therefore unlikely to be a primary defect predisposing non-diabetic individuals or patients with NIDDM for the development of CHD. This does not dispute a role for insulin resistance per se in being a risk factor for cardiovascular disease but suggests that the mechanisms linking the two phenomena are likely to be indirect or could involve some other insulin sensitive process(es) such as resistance to the platelet antiaggregating effect of insulin, which also characterizes patients with NIDDM [32].

Acknowledgements. Supported by grants from the Academy of Finland (H. Y.), the Novo Nordisk (H. Y., P.N.), Sigrid Juselius (H. Y.), and Ahokas (H.Y., P.N.) foundations, the Research
Foundation of Orion Corporation (T.U.) and the M.D./Ph.D. Program of the University of Helsinki (T.U.).

\section{References}

1. Fuller JH, Shipley MJ, Rose G, Jarrett RJ, Keen H (1980) Coronary-heart-disease risk and impaired glucose tolerance. The Whitehall Study. Lancet 1(8183):1371-1376

2. Donahue RP, Orchard TJ (1992) Diabetes mellitus and macrovascular complications. An epidemiological perspective. Diabetes Care 15: 1141-1155

3. Warram JH, Martin BC, Krolewski AS, Soeldner JS, Kahn R (1990) Slow glucose removal rate and hyperinsulinemia precede the development of type II diabetes in the offspring of diabetic parents. Ann Intern Med 113: 909-915

4. Pyörälä K (1979) Relationship of glucose tolerance and plasma insulin to the incidence of coronary heart disease: results from two population studies in Finland. Diabetes Care 2: 131-141

5. Austin MA, Mykkänen L, Kuusisto J, et al. (1995) Prospective study of small LDLs as a risk factor for non-insulin dependent diabetes mellitus in elderly men and women. Circulation 92: 1770-1778

6. Després J-P, Lamarche B, Mauriège P, et al. (1996) Hyperinsulinaemia as an independent risk factor for ischaemic heart disease. N Engl J Med 334: 952-957

7. Paternostro G, Camici PG, Lammertsma AA, et al. (1996) Cardiac and skeletal muscle insulin resistance in patients with coronary heart disease. A study with positron emission tomography. J Clin Invest 98: 2094-2099

8. Voipio-Pulkki L-M, Nuutila P, Knuuti MJ, et al. (1993) Heart and skeletal muscle glucose disposal in type 2 diabetic patients as determined by positron emission tomography. J Nucl Med 34: 2064-2067

9. Scheuer J (1993) Metabolic factors in myocardial failure. Circulation 87 [Suppl. VII]:VII-54-VII-57

10. Mokan M, Gerich JE (1992) A simple insulin infusion algorithm for establishing and maintaining overnight near-normoglycemia in type I and type II diabetes. J Clin Endocrinol Metab 74: 943-945

11. DeFronzo RA, Tobin JD, Andres R (1979) Glucose clamp technique: a method for quantifying insulin secretion and resistance. Am J Physiol 237:E214-E223

12. Nuutila P, Koivisto VA, Knuuti J, et al. (1992) Glucose-free fatty acid cycle operates in human heart and skeletal muscle in vivo. J Clin Invest 89: 1767-1774

13. Schelbert HR, Henze E, Schon HR, et al. (1983) C-11 palmitate for the noninvasive evaluation of regional myocardial fatty acid metabolism with positron computed tomography. III. In vivo demonstration of the effects of substrate availability on myocardial metabolism. Am Heart J 105: 492-504

14. Nuutila P, Knuuti MJ, Heinonen OJ, et al. (1994) Different alterations in the insulin-stimulated glucose uptake in the athlete's heart and skeletal muscle. J Clin Invest 93: 2267-2274

15. Devereux RB, Alonso DR, Lutas EM, et al. (1986) Echocardiographic assessment of left ventricular hypertrophy: comparison to necropsy findings. Am J Cardiol 57: 450-458

16. Krivokapich J, Huang S-C, Selin CE, Phelps ME (1987) Fluorodeoxyglucose rate constants, lumped constant, and glucose metabolic rate in rabbit heart. Am J Physiol 252:H777-H787

17. Ratib O, Phelps ME, Huang S-C, Henze E, Selin CE, Schelbert HR (1982) Positron tomography with deoxyglu- 
cose for estimating local myocardial glucose metabolism. $\mathbf{J}$ Nucl Med 23: 577-586

18. Hariharan R, Bray M, Ganim R, et al. (1995) Fundamental limitations of $\left[{ }^{18} \mathrm{~F}\right] 2$-deoxy-2-fluoro-D-glucose for assessing myocardial glucose uptake. Circulation 91: 2435-2444

19. Mäki M, Luotolahti M, Nuutila P, et al. (1996) Glucose uptake in the chronically dysfunctional but viable myocardium. Circulation 93: 1658-1666

20. Monti LD, Lucignani G, Landoni C, et al. (1995) Myocardial glucose uptake evaluated by positron emission tomography and fluorodeoxyglucose during hyperglycemic clamp in IDDM patients. Diabetes 44: 537-542

21. Ferrannini E, Santoro D, Bonadonna R, Natali A, Parodi O, Camici PG (1993) Metabolic and hemodynamic effects of insulin on human hearts. Am J Physiol 264: E308-E315

22. Lassers BW, Kaijser L, Carlson LA (1972) Myocardial lipid and carbohydrate metabolism in healthy, fasting men at rest: studies during continuous infusion of ${ }^{3} \mathrm{H}$-palmitate. Eur J Clin Invest 2: 348-358

23. Nuutila P, Knuuti J, Ruotsalainen U, et al. (1993) Insulin resistance is localized to skeletal but not heart muscle in type 1 diabetes. Am J Physiol 264: E756-E762

24. vom Dahl J, Herman WH, Hicks RJ, et al. (1993) Myocardial glucose uptake in patients with insulin-dependent diabetes mellitus assessed quantitatively by dynamic positron emission tomography. Circulation 88: 395-404
25. Nuutila P, Mäki M, Laine H, et al. (1995) Insulin action on heart and skeletal muscle glucose uptake in essential hypertension. J Clin Invest 96: 1003-1009

26. Thomas JA, Marks BH (1978) Plasma norepinephrine in congestive heart failure. Am J Cardiol 41: 233-243

27. Paolisso G, De Riu S, Marrazzo G, Verza M, Varricchio M, D'Onofrio F (1991) Insulin resistance and hyperinsulinaemia in patients with chronic congestive heart failure. Metabolism 40: 972-977

28. Ng CK, Holden JE, DeGrado TR, Raffel DM, Kornguth ML, Gatley SJ (1991) Sensitivity of myocardial fluorodeoxyglucose lumped constant to glucose and insulin. Am J Physiol 260: H593-H603

29. Nurjhan N, Campell PJ, Kennedy FP, Miles JM, Gerich JE (1986) Insulin dose-response characteristics for suppression of glycerol release and conversion to glucose in humans. Diabetes 35: 1326-1331

30. Groop LC, Bonadonna RC, Del Prato S, et al. (1989) Glucose and free fatty acid metabolism in non-insulin-dependent diabetes mellitus. Evidence for multiple sites of insulin resistance. J Clin Invest 84: 205-213

31. Nuutila P, Knuuti MJ, Raitakari M, et al. (1994) Effect of antilipolysis on heart and skeletal muscle glucose uptake in overnight fasted humans. Am J Physiol 267: E941-E946

32. Trovati M, Mularoni E, Burzacca S, et al. (1995) Impaired insulin-induced platelet antiaggregating effect in obesity and in obese NIDDM patients. Diabetes 44: 1318-1322 